\title{
Mid-late pregnancy maternal glycolipids in gestational diabetes mellitus (GDM) fluctuated in glycosylated hemoglobin levels of $5.5 \%-6.4 \%$ associated with umbilical cord blood of amino acid and carnitine profiles: a nested case- cohort observational study
}

long yuhang

Sun Yat-Sen University https://orcid.org/0000-0002-5751-805X

Le Chen

Sun Yat-Sen University

Yaochao Yang

Sun Yat-Sen University

Wei Liu

Sun Yat-Sen University

Hanxi Zhang

Chinese Academy of Medical Sciences \& Peking Union Medical College Fuwai Hospital

WuJuan Chen

Sun Yat-Sen University

Fangfang Jiang

Sun Yat-Sen University

Ting Hong

Sun Yat-Sen University

Jianying Wang

Sun Yat-Sen University

Yao Chen

Sun Yat-Sen University

Jianyong Cao

Sun Yat-Sen University

Dan Xie

Sun Yat-Sen University

Zhaojuan Su ( $\sim$ 2847066683@qq.com )

The Fifth Affiliated Hospital of Sun Yat-Sen University https://orcid.org/0000-0003-2633-1556 
Research article

Keywords: GDM, amino acid, carnitine

Posted Date: June 30th, 2020

DOI: https://doi.org/10.21203/rs.3.rs-36779/v1

License: (c) (i) This work is licensed under a Creative Commons Attribution 4.0 International License. Read Full License 


\section{Abstract \\ Background}

To explore the relationship between maternal-neonatal clinical data and the changes of cord blood amino acid and carnitine levels between pregnant women with gestational diabetes mellitus (GDM) when glycosylated hemoglobin levels of 5.5\%-6.4\% excluded diabetic medicine as GDM ${ }^{a}$ group and the control group.

\section{Methods}

In all, 312 qualified participants were recruited with permission from the ethical department of the Fifth Affiliated Hospital of Sun Yat-Sen University and divided into two groups (GDM ${ }^{\mathrm{a}}$ and non-GDM) after adjusting for maternal age and body mass index (BMI) between the 1 June 2017 and 30 April 2020. All maternal-neonatal data were collected and analysed at the centre.

\section{Results}

Interestingly, glycine in cord blood was not only significantly different between groups(594.19 vs 407.45, $\mathrm{P}<0.001$ ), but also associated among those pregnant women in GDM when glycosylated hemoglobin levels of $5.5 \%-6.4 \%$ through the standard of diet control and exercise $(b=0.449, P=0.010)$. Meanwhile, neonatal hypoglycemia had the positive association with GDM monitoring glycosylated hemoglobin levels of $5.5 \%-6.4 \%(P=0.031$, OR $5.7795 \% \mathrm{CI}[1.18,28.36])$, but none of correlations with umbilical cord blood of amino acids and carnitines.

\section{Conclusions}

The study identifies some differences and relationships in maternal-neonatal data only when GDM ${ }^{a}$ group, glycosylated hemoglobin levels of $5.5 \%-6.4 \%$ without diabetic medicine, compared with the control group which adjusted by age and BMI. Particularly, umbilical cord blood of glycine levels is related to the diabetic status of glycosylated hemoglobin $5.5 \%-6.4 \%$ in GDM during pregnancy and could be potential diabetic mechanism by maternal-fetal interface.

\section{Background}

Gestational diabetes mellitus (GDM), defined as a common and particular disease because of its own effects on other adverse pregnancy outcomes, such as hypertensive disorders complicating pregnancy, foetal growth disorders, caesarean delivery, postoperative complications and wound infections, is widely accepted as affecting the entire pregnancy process and constituting a major risk factor for perinatal 
complications ${ }^{[1,2]}$. In China, approximately $17.5 \%$ of pregnant women with GDM are diagnosed by an abnormal 75-g oral glucose tolerance test (OGTT) at 24-28 weeks' gestation according to the new screening criteria recommended by the International Association of Diabetes and Pregnancy Study Groups (IADPSG) ${ }^{[3]}$ based on the hyperglycaemia and adverse pregnancy outcomes (HAPO) study ${ }^{[4]}$.

GDM, out of control blood glucose levels as an obvious dysglycaemia, is associated with an increased risk of adverse pregnancy outcomes, such as neonatal hypoglycaemia ${ }^{[5,6]}$. Numerous metabolites correlated with insulin resistance and dysglycaemia have been elucidated by recent metabolomics studies $[7,8]$. Foetal metabolites associated with dysglycaemia include carbohydrates ${ }^{[9]}$, lipid profiles ${ }^{[10]}$, carnitines ${ }^{[11]}$ and amino acids ${ }^{[12]}$, particularly branched-chain amino acids ${ }^{[13]}$ and aromatic amino acids ${ }^{[14]}$. In addition, maternal dyslipidaemia may be associated with neonatal metabolism changes, such as amino acid metabolism and carnitine levels ${ }^{[12]}$. Neonate-related metabolic changes are generally associated with increased maternal glycometabolism and lipid metabolism ${ }^{[15]}$, and it has been found that maternal lipid levels increase during the entire pregnancy, which is similar in non-GDM women or GDM women ${ }^{[16,17]}$. Pregnancy is a unique metabolic state that is characterized by progressive increases in circulating umbilical cord amino acids and carnitines to optimize the availability of substrates necessary for foetal development ${ }^{[18]}$.

Furthermore, maternal glycolipid metabolism tends to remain elevated because of the lipolytic effects of placental hormones and insulin resistance, which occur particularly in mid-late pregnancy $[19,20]$. Maternal dysglycaemia and dyslipidaemia have been demonstrated to be associated with abnormal foetal growth, such as macrosomia ${ }^{[21]}$. However, the exact effect of how maternal glycolipid metabolism changes amino acid metabolism and carnitine levels in newborns remains unclear. Therefore, we carried out a nested case-cohort observational study to explore the associations between maternal glycolipid levels during mid-late pregnancy and levels of neonatal amino acids and carnitines stratified into two groups, GDM and non-GDM, adjusting for age and antepartum body mass index (BMI). It will provide effective strategies for the prevention of adverse pregnant outcomes and a helpful method for understanding the mechanism of maternal and neonatal metabolism.

\section{Methods}

\subsection{Ethics statement}

The present study has been approved by the ethical committee of the Fifth Affiliated Hospital of Sun YatSen University, and all participants provided written informed consent.

\subsection{Study population}

Singleton pregnant women who received regular prenatal care during their entire pregnancy; took blood tests including OGTT and glycated haemoglobin from 24-28 weeks' gestation and antepartum glycated haemoglobin and lipid tests such as triglycerides (TG), total cholesterol (TC), high-density lipoprotein- 
cholesterol (HDL-C), low-density lipoprotein-cholesterol (LDL-C), small and dense low-density lipoproteincholesterol (sdLDL-C) and non-high-density lipoprotein-cholesterol (non-HDL-C); and delivered in our centre between 1 June 2017 and 30 April 2020 were recruited for the study population. All cases were divided into 156 GDM patients with paired non-GDM patients adjusting for age and BMI (Fig. 1 \& Appendix Fig. 1). Those glycosylated hemoglobin levels of 5.5\%-6.4\% in GDM group was defined as $\mathrm{GDM}^{\mathrm{a}}$ group.Umbilical cord blood was collected and analysed for 17 types of amino acids and 30 types of carnitines after the delivery of the placenta.

\subsection{Diagnosis standards and medical care}

The diagnosis of GDM was based on a 75-g oral glucose tolerance test (OGTT) performed between 24 and 28 gestational weeks, according to the American Diabetes Association (ADA) criteria (fasting $\geq$ $5.1 \mathrm{mmol} / \mathrm{L}, 1 \mathrm{~h} \geq 10.0 \mathrm{mmol} / \mathrm{L}, 2 \mathrm{~h} \geq 8.5 \mathrm{mmol} / \mathrm{L}$ ). Recruited patients accepted the standard of treatment for GDM according to the American College of Obstetricians and Gynecologists (ACOG) practice bulletin on gestational diabetes mellitus.

The diagnosis of neonatal hypoglycemia was based on fingertip blood glucose test after the labour within 5 minutes, according to the American Academy of Pediatrics criteria (fingertip blood glucose < $2.22 \mathrm{mmol} / \mathrm{L})^{[22]}$.

\subsection{Clinical data}

Clinical data, including age, gestational weeks, BMl, weight gain, mode of delivery, foetal length, birth weight, and head circumference, were obtained from subjects or measured by researchers.

\subsection{Blood sample collection}

In brief, all pregnant women had blood tests from the prenatal examination or hospitalization examination before their delivery. All neonates included in our study had amino acids and carnitines measured in dried blood specimens collected from cord blood at our centres after the labour of placenta within 10 minutes.

\subsection{Laboratory measurements}

\section{Maternal blood test}

Samples in the anti-coagulative tubes for measuring glycated haemoglobin, lipid profiles and glucose in plasma were sent to the clinical laboratory within $1 \mathrm{~h}$ of collection. The other samples were placed at room temperature $\left(25^{\circ} \mathrm{C}\right)$ for $1 \mathrm{~h}$ to allow the blood to clot. After blood coagulation, sera were separated by centrifugation at $720 \mathrm{~g}$ for $15 \mathrm{~min}$ at $4{ }^{\circ} \mathrm{C}$, frozen in aliquots of $100 \mathrm{~mL}$, and stored at $-80^{\circ} \mathrm{C}$ until analysis.

Plasma glucose levels were measured using a GOD-PAP kit (Human, Shanghai, China). Glycated haemoglobin was tested by an ADAMS ${ }^{\text {TM }}$ A1c HA-8180 kit (Arkrayusa Inc, Kyoto, Japan). Triglyceride (TG) 
levels were measured using a glycerol phosphate oxidase test kit (Shanghai, China). Total cholesterol (TC) levels were measured using an oxidase test kit (Human, Shanghai, China). High-density lipoproteincholesterol (HDL-C) and low-density lipoprotein-cholesterol (LDL-C) levels were determined by direct method kits (Human, Shanghai, China). Non-high-density lipoprotein-cholesterol (non-HDL-C) was calculated as TC minus HDL-C. Furthermore, small and dense low-density lipoprotein cholesterol levels were measured using an immunoturbidimetric assay kit (BSBE CO., Beijing, China).

\section{Cord blood test}

One single 3.2-mm-diameter dried cord blood disc per sample was punched from the dried blood spot card and extracted with 90 ul of methanol (including internal standards from Cambridge Isotope Inc.) at $30{ }^{\circ} \mathrm{C}$ for 30 minutes. After centrifugation at $2000 \mathrm{~g}$ for 5 minutes, $50 \mathrm{ul}$ of supernatant was transferred and nitrogen-dried at room temperature, followed by addition of $50 \mathrm{ul}$ of derivatization reagent (acetylchloride: butanol (v:v) =1:9), incubation at $60^{\circ} \mathrm{C}$ for 30 minutes, nitrogen-drying, and reconstitution with $75 \mathrm{ul}$ of $80 \%$ acetonitrile/ $\mathrm{H}_{2} \mathrm{O}(\mathrm{v} / \mathrm{v})$. After centrifugation at $4000 \mathrm{~g}$ for 15 minutes, $50 \mathrm{ul}$ of supernatant was taken for liquid chromatographic-mass spectrometric (LC-MS) analysis.

An Acquity UPLC I-Class Xevo TQD mass spectrometer (Waters) was used to measure the concentration levels of multiple amino acids and carnitines in the dried blood spot with the application of direct flow injection and multiple reaction monitoring (MRM). The sample was introduced into the electrospray ionization interface by the mobile phase of $80 \%$ acetonitrile $/ \mathrm{H}_{2} \mathrm{O}(\mathrm{v} / \mathrm{v})$ with scheduled flow rates. The mass spectrometer was tuned as recommended by the manufacturer with resolution, capillary voltage, source temperature, and desolvation temperature set at $0.7 \mathrm{Da}, 3.5 \mathrm{kV}, 120^{\circ} \mathrm{C}$, and $350^{\circ} \mathrm{C}$, respectively. All the targeted analytes were quantitatively measured according to the ion pair transitions of both their own and corresponding internal standards. Masslynx NT 4.1 Software Suite was used to control the instrument and for data processing and analysis.

\subsection{Statistical analysis}

Continuous variables and categorical variables are presented as the median (10th, 90th percentile) or mean (SD) and counts (percentage) as appropriate. Differences between two groups were analysed with the Mann-Whitney $\mathrm{U}$ test or chi-square test. Correlations between maternal data or neonatal clinical data and foetal cord blood test indexes were analysed by the Spearman rank correlation test. Significance was assumed at $P<0.05$. Then, Multivariable logistic regression analyses were performed to assess the adjusted association between maternal variables and neonatal hypoglycemia. Meanwhile, an ANCOVA analysis was performed to study the relationship between umbilical cord blood of amino acid and carnitine by adjusting other cofactors. Statistical analysis was performed with SPSS for Windows software (version 22, SPSS, IBM, New York, NY).

\section{Results}

\subsection{Baseline characteristics}


Follow-up with the study population was adjusted by maternal age and BMI. Table 1 shows the baseline characteristics of the 312 study participants of those who developed GDM and extracted randomly controls (156 cases and 156 non-cases) from a nested case-cohort study framework (Figure 1 \& Appendix Figure 1). Compared with non-cases, participants who developed GDM monitoring glycosylated hemoglobin levels of $5.5 \%-6.4 \%$ were more likely to have had higher incidence of hypoglycemia in neonates but less gestational weeks.

\subsection{Comparison of maternal and foetal blood tests between groups}

Considering the maternal blood test levels at 24-28 gestational weeks, all maternal blood glucose test results in the GDMa group were higher than those in the non-GDM group. TG and non-HDL-C levels showed a slight increase in the GDM ${ }^{a}$ group compared with the non-GDM group in the antepartum period (Table 2).

The baseline concentrations of each of the individual carnitine species and amino acids from cord blood are presented in Table 2. Only glycine levels (594.19 vs 407.45, $\boldsymbol{P}<0.001$ ) in cord blood were significantly different between groups. Meanwhile, two long-chain carnitines including isovalerylcarnitine $(\boldsymbol{P}=0.019)$ and 3-hydroxyisovaleryl-carnitine $(\boldsymbol{P}=0.037)$ were different between groups.

\subsection{Association between maternal-foetal clinical data and blood tests}

Our data shows that neonatal hypoglycemia were positively correlated with antepartum BMI $(\boldsymbol{r}=0.126, \boldsymbol{P}$ $=0.027)$, GDM in glycosylated hemoglobin monitoring 5.5-6.4\% $(\boldsymbol{r}=0.217, \boldsymbol{P}<0.001)$, glycosylated hemoglobin before delivery $(\boldsymbol{r}=0.148, \boldsymbol{P}=0.009)$ but negatively correlated with gestational weeks $(\boldsymbol{r}=-0.119, \boldsymbol{P}=0.036)$ (Table 3). Then, Table 5 summarizes the association between the maternal demographic variables and neonatal hypoglycemia analyzed with a multivariate regression. Only GDM when glycosylated hemoglobin was the range between $5.5 \%$ and $6.4 \%$ are associated with neonatal hypoglycemia $(\boldsymbol{P}=0.031, O R 5.7795 \% \boldsymbol{C}\{1.18,28.36])$, but antepartum BMI, glycosylated hemoglobin before delivery and gestational weeks was not.

In the other hand, cord blood glycine, isovalerylcarnitine and 3-hydroxyisovaleryl-carnitine levels were all positively correlated with $\mathrm{GDM}^{\mathrm{a}}$ and glycosylated hemoglobin (all $\boldsymbol{P}<0.05$ ). Additionally, maternal gestational weeks only had negative correlations with glycine and isovalerylcarnitine, and the positive correlation between glycine and antepartum BMI. However, none of umbilical cord blood of amino acid and carnitine had the relationship with neonatal hypoglycemia (Table 4).

Table 6 shows an ANCOVA analysis that the relationship between cord blood glycine, isovalerylcarnitine and 3-hydroxyisovaleryl-carnitine levels and maternal data. However, only cord blood glycine levels were elevated in $\operatorname{GDM}^{\mathrm{a}}(\boldsymbol{b}=0.449, \boldsymbol{P}=0.010)$

\section{Discussion}


Foetal metabolism, as indicated by carnitine levels and amino acids, is related to the risk of preterm infants, maternal gestational age and neonatal development ${ }^{[23,24]}$. Dysglycaemia is an important link between maternal serum amino acids and carnitines ${ }^{[25]}$, maternal obesity/overweight ${ }^{[26]}$ and maternal and foetal lipid profiles ${ }^{[27]}$. We explored the relationship between GDM fluctuated in glycosylated hemoglobin levels of $5.5 \%-6.4 \%\left(\right.$ as GDM $\left.^{a}\right)$ and neonatal carnitine levels and amino acids and their potential influence on foetal growth. The result of the present study showed that, although GDMa was not correlated with any neonatal carnitine levels, it was positively associated with glycine. Some studies show that plasma glycine correlates positively with glucose disposal and dietary glycine supplementation increases insulin ${ }^{[28]}$. Glycine levels rises higher in $\mathrm{GDM}^{\mathrm{a}}$ group than the control group and the elevation of insulin levels probably could be followed to contribute neonatal hypoglycemia. However, none of correlation on foetal glycine levels with neonatal hypoglycemia.

The crosstalk between GDM and foetal growth is well established ${ }^{[29]}$. During pregnancy, dysglycaemia is typically accompanied by dyslipidaemia ${ }^{[30]}$, and together they promote an adverse metabolic intrauterine environment and lead to preterm infants ${ }^{[31]}$ or congenital malformations ${ }^{[32]}$. GDM is strongly correlated with foetal growth ${ }^{[33]}$, but whether GDM can influence foetal carnitine levels is unclear.

In our study, there were some significant differences between groups for the measured amino acid parameters in newborns after adjusting for maternal age and BMI. Surprisingly, cord blood glycine was not only different between groups but was also correlated with GDM when glycosylated hemoglobin levels of $5.5 \%-6.4 \%$. However, neonatal carnitine levels were not associated with GDM ${ }^{a}$ in the present study.

Foetal development is influenced by neonatal and maternal metabolism [ ${ }^{[3,35]}$, and in the present study, we analysed the effects of neonatal carnitine levels and amino acids on neonatal growth parameters. We found that cord blood glycine levels were correlated with GDMa ${ }^{a}$. Maternal TG were different between two groups but was not associated with neonatal carnitine levels and amino acids.

The association between maternal glycolipid metabolism and neonatal development has been reported in previous studies [36], and our results support these studies in a much larger study population. Birth weight and foetal length have been shown to be positively correlated with carnitine levels in cord blood in previous studies ${ }^{[37]}$. However, the negative associations between carnitine levels in neonates. The results in the present study not only found that glycine levels were positively associated with maternal glycometabolism but also firstly revealed the association between maternal lipid profile levels in late gestation and neonatal carnitine levels and amino acids in a new research population.

There are several limitations in the present study. First, although the present study provided insight into the association between maternal glycolipid factors and neonatal metabolism and characteristic size, the retrospective design could be seen as another limitation. Additionally, since insulin levels of cord blood in the present case-cohort was not check in the two groups, the relationship between glycine and the 
function of insulin including C-peptide was unclear. Furthermore, variations in lipid concentration are considerable during gestation, but we only obtained lipid values in late gestation. Therefore, further prospective studies measuring maternal glycolipid concentrations across multiple gestational times in a wide-ranging population are needed to increase the validity of the results.

\section{Conclusion}

In summary, our results show that glycine levels in cord blood were strongly increased in the GDM group which fluctuated in glycosylated hemoglobin levels of $5.5 \%-6.4 \%$, compared to the non-GDM group after adjusting for maternal age and BMI. Meanwhile, glycine levels had a positive relationship with GDM in glycosylated hemoglobin levels of 5.5\%-6.4\%, but was not correlated with neonatal hypoglycemia. Maternal TG levels in late pregnancy are different in two groups, but there were no correlation with umbilical cord blood of amino acid and carnitine profiles in our study. Elucidation of the underlying mechanisms of maternal glycolipid levels are needed to place these results in a clinical perspective for neonatal hypoglycemia and development.

\section{Abbreviations}

\begin{tabular}{|l|l|}
\hline gestational diabetes mellitus & GDM \\
\hline body mass index & BMI \\
\hline oral glucose tolerance test & OGTT \\
\hline International Association of Diabetes and Pregnancy Study Groups & IADPSG \\
\hline hyperglycaemia and adverse pregnancy outcomes & HAPO \\
\hline triglycerides & TG \\
\hline total cholesterol & TC \\
\hline high-density lipoprotein-cholesterol & HDL-C \\
\hline low-density lipoprotein-cholesterol & LDL-C \\
\hline small and dense low-density lipoprotein-cholesterol & sdLDL-C \\
\hline non-high-density lipoprotein-cholesterol & non-HDL-C \\
\hline American Diabetes Association & ADA \\
\hline American College of Obstetricians and Gynecologists & ACOG \\
\hline
\end{tabular}

\section{Declarations}

Ethics approval and consent to participate:

The study protocol was established, according to the ethical guidelines of the Helsinki Declaration and was approved by the ethical committee of the Fifth Affiliated Hospital of Sun Yat-Sen University.

Consent for publication: 
Written informed consent was obtained from individual or guardian participants.

Availability of data and material:

All data generated or analyzed in the study are included in this published article.

Competing interests:

The authors declare that they have no competing interests. No conflicts of interest that could be perceived as prejudicing the impartiality of the research are reported.

Funding:

This project was funded by the Science and Technology Project of China, Zhuhai (to ZS). The funding organisation had no role in the study design, data collection, analysis, interpretation, or manuscript writing.

Authors' contributions

$Y L$ and $Z S$ designed experiments; $Y Y, W L$ and $L C$ carried out experiments; $Y L, H Z$ and $W C$ analyzed experimental results. YY, YL, LC and $\mathrm{WL}$ analyzed sequencing data and developed analysis tools. FJ, TH, JW, YC and JC made a series of inclusion and follow-up of patients. DX randomly extracted samples to match. YL wrote the manuscript.

Acknowledgements:

We appreciated Ran Xue for the analysis of Cord blood test on the manuscript.

\section{References}

1. Senat MV, Affres H, Letourneau A, Coustols-Valat M, Cazaubiel M, Legardeur H, et al. Effect of Glyburide vs Subcutaneous Insulin on Perinatal Complications Among Women With Gestational Diabetes: A Randomized Clinical Trial. JAMA. 2018;319(17):1773-80.

2. Chiefari E, Arcidiacono B, Foti D, Brunetti A. Gestational diabetes mellitus: an updated overview. J Endocrinol Invest. 2017;40(9):899-909.

3. Gerome JM, Bucher LKM, Dogbey G. Effects of Implementing International Association of Diabetes and Pregnancy Study Groups Gestational Diabetes Screening on Pregnancy Outcomes at a Small Community Teaching Hospital. Clin Diabetes. 2017;35(2):84-9.

4. Lowe LP, Metzger BE, Dyer AR, Lowe J, McCance DR, Lappin TR, et al. Hyperglycemia and Adverse Pregnancy Outcome (HAPO) Study: associations of maternal A1C and glucose with pregnancy outcomes. Diabetes Care. 2012;35(3):574-80.

5. Wang C, Wei Y, Zhang X, Zhang Y, Xu Q, Sun Y, et al. A randomized clinical trial of exercise during pregnancy to prevent gestational diabetes mellitus and improve pregnancy outcome in overweight 
and obese pregnant women. Am J Obstet Gynecol. 2017;216(4):340-51.

6. Tieu J, McPhee AJ, Crowther CA, Middleton P, Shepherd E. Screening for gestational diabetes mellitus based on different risk profiles and settings for improving maternal and infant health. Cochrane Database Syst Rev. 2017;8:CD007222.

7. Adeva-Andany M, Souto-Adeva G, Ameneiros-Rodriguez E, Fernandez-Fernandez C, Donapetry-Garcia C, Dominguez-Montero A. Insulin resistance and glycine metabolism in humans. Amino Acids. 2018;50(1):11-27.

8. Strand E, Rebnord EW, Flygel MR, Lysne V, Svingen GFT, Tell GS, et al. Serum Carnitine Metabolites and Incident Type 2 Diabetes Mellitus in Patients With Suspected Stable Angina Pectoris. J Clin Endocrinol Metab. 2018;103(3):1033-41.

9. Ackerman DL, Craft KM, Townsend SD. Infant food applications of complex carbohydrates: Structure, synthesis, and function. Carbohydr Res. 2017;437:16-27.

10. Geraghty AA, Alberdi G, O'Sullivan EJ, O'Brien EC, Crosbie B, Twomey PJ, et al. Maternal and fetal blood lipid concentrations during pregnancy differ by maternal body mass index: findings from the ROLO study. BMC Pregnancy Childbirth. 2017;17(1):360.

11. Tajima G, Hara K, Tsumura M, Kagawa R, Okada S, Sakura N, et al. Newborn screening for carnitine palmitoyltransferase II deficiency using (C16 + C18:1)/C2: Evaluation of additional indices for adequate sensitivity and lower false-positivity. Mol Genet Metab. 2017;122(3):67-75.

12. Liu G, Deng W, Cui W, Xie Q, Zhao G, Wu X, et al. Analysis of amino acid and acyl carnitine profiles in maternal and fetal serum from preeclampsia patients. The journal of maternal-fetal \& neonatal medicine: the official journal of the European Association of Perinatal Medicine, the Federation of Asia and Oceania Perinatal Societies, the International Society of Perinatal Obstet. 2018:1-151.

13. Manta-Vogli PD, Schulpis KH, Dotsikas Y, Loukas YL. The significant role of amino acids during pregnancy: nutritional support. The Journal of Maternal-Fetal \& Neonatal Medicine. 2018:1-7.

14. Edward A. Liechty DWB, Moorehead H, Auble L, Scott C. Denne. Aromatic amino acids are utilized and protein synthesis is stimulated during amino acid infusion in the ovine fetus. Nutrient Metabolism. 1999;129(6):1161-6.

15. Ryckman KK, Spracklen CN, Smith CJ, Robinson JG, Saftlas AF. Maternal lipid levels during pregnancy and gestational diabetes: a systematic review and meta-analysis. BJOG. 2015;122(5):643-51.

16. Vidakovic AJ, Jaddoe VW, Voortman T, Demmelmair H, Koletzko B, Gaillard R. Maternal plasma polyunsaturated fatty acid levels during pregnancy and childhood lipid and insulin levels. Nutr Metab Cardiovasc Dis. 2017;27(1):78-85.

17. Martin CL, Vladutiu CJ, Zikry TM, Grace MR, Siega-Riz AM. Maternal lipid levels during pregnancy and child weight status at 3 years of age. Pediatr Obes. 2018:e12485.

18. Liu J, Chen XX, Li XW, Fu W, Zhang WQ. Metabolomic Research on Newborn Infants With Intrauterine Growth Restriction. Med (Baltim). 2016;95(17):e3564. 
19. Heazell AE, Bernatavicius G, Warrander L, Brown MC, Dunn WB. A metabolomic approach identifies differences in maternal serum in third trimester pregnancies that end in poor perinatal outcome. Reproductive sciences. 2012;19(8):863-75.

20. Huang S, Mo TT, Norris T, Sun S, Zhang T, Han TL, et al. The CLIMB (Complex Lipids In Mothers and Babies) study: protocol for a multicentre, three-group, parallel randomised controlled trial to investigate the effect of supplementation of complex lipids in pregnancy, on maternal ganglioside status and subsequent cognitive outcomes in the offspring. BMJ Open. 2017;7(10):e016637.

21. Azar M, Stoner JA, Dao HD, Stephens L, Goodman JR, Maynard J, et al. Epidemiology of Dysglycemia in Pregnant Oklahoma American Indian Women. J Clin Endocrinol Metab. 2015;100(8):2996-3003.

22. Committee onF, Newborn, Adamkin DH. Postnatal glucose homeostasis in late-preterm and term infants. Pediatrics. 2011;127(3):575-9.

23. Bonsante F, Gouyon JB, Robillard PY, Gouyon B, lacobelli S. Early optimal parenteral nutrition and metabolic acidosis in very preterm infants. PLoS One. 2017;12(11):e0186936.

24. Clark MA, Stein REK, Silver EJ, Khalid S, Fuloria M, Esteban-Cruciani NV. Carnitine deficiency in preterm infants: A national survey of knowledge and practices. J Neonatal Perinatal Med. 2017;10(4):381-6.

25. Nevalainen J, Sairanen M, Appelblom H, Gissler M, Timonen S, Ryynanen M. First-Trimester Maternal Serum Amino Acids and Acylcarnitines Are Significant Predictors of Gestational Diabetes. Rev Diabet Stud. 2016;13(4):236-45.

26. De Souza LR, Berger H, Retnakaran R, Maguire JL, Nathens AB, Connelly PW, et al. First-Trimester Maternal Abdominal Adiposity Predicts Dysglycemia and Gestational Diabetes Mellitus in Midpregnancy. Diabetes Care. 2016;39(1):61-4.

27. Herrera E, Desoye G. Maternal and fetal lipid metabolism under normal and gestational diabetic conditions. Horm Mol Biol Clin Investig. 2016;26(2):109-27.

28. Yan-Do R, MacDonald PE. Impaired "Glycine"-mia in Type 2 Diabetes and Potential Mechanisms Contributing to Glucose Homeostasis. Endocrinology. 2017;158(5):1064-73.

29. Goldstein RF, Abell SK, Ranasinha S, Misso M, Boyle JA, Black MH, et al. Association of Gestational Weight Gain With Maternal and Infant Outcomes: A Systematic Review and Meta-analysis. JAMA. 2017;317(21):2207-25.

30. Herrera Martinez A, Palomares Ortega R, Bahamondes Opazo R, Moreno-Moreno P, Mf MP, GalvezMoreno MA. Hyperlipidemia during gestational diabetes and its relation with maternal and offspring complications. Nutr Hosp. 2018;35(3):698-706.

31. Zheng W, Huang W, Zhang L, Tian Z, Wang T, Zhang T, et al. Changes in Serum Lipid Levels During Pregnancy and Association With Neonatal Outcomes: A Large Cohort Study. Reproductive sciences. 2018;25(9):1406-12.

32. Bequer L, Gomez T, Molina JL, Alvarez A, Chaviano C, Clapes S. Experimental diabetes impairs maternal reproductive performance in pregnant Wistar rats and their offspring. Syst Biol Reprod Med. 
2018;64(1):60-70.

33. Johns EC, Denison FC, Norman JE, Reynolds RM. Gestational Diabetes Mellitus: Mechanisms, Treatment, and Complications. Trends Endocrinol Metab. 2018;29(11):743-54.

34. Akkaya H, Buke B, Uysal G. Fetal pancreatic hyperechogenicity may be an early ultrasonographic sign of gestational diabetes mellitus. The journal of maternal-fetal \& neonatal medicine: the official journal of the European Association of Perinatal Medicine, the Federation of Asia and Oceania Perinatal Societies, the International Society of Perinatal Obstet. 2018:1-121.

35. Barbour LA, Hernandez TL. Maternal Lipids and Fetal Overgrowth: Making Fat from Fat. Clin Ther. 2018;40(10):1638-47.

36. Lowe WL Jr, Scholtens DM, Lowe LP, Kuang A, Nodzenski M, Talbot O, et al. Association of Gestational Diabetes With Maternal Disorders of Glucose Metabolism and Childhood Adiposity. JAMA. 2018;320(10):1005-16.

37. Lowe WL Jr, Bain JR, Nodzenski M, Reisetter AC, Muehlbauer MJ, Stevens RD, et al. Maternal BMI and Glycemia Impact the Fetal Metabolome. Diabetes Care. 2017;40(7):902-10.

\section{Tables}




\begin{tabular}{|c|c|c|c|c|}
\hline & $\mathrm{GDM}^{\mathrm{a}}$ & non-GDM & $c 2$ & $P$ \\
\hline $\mathrm{N}$ & 156 & 156 & & \\
\hline Maternal age\years $\square$ & $32.79 \pm 3.82$ & $33.46 \pm 3.80$ & - & 0.098 \\
\hline Gestational weeks $\square$ days $\square$ & $271.13 \pm 8.38$ & $275.77 \pm 9.78$ & - & $<0.001 *$ \\
\hline \multicolumn{5}{|l|}{ Body mass index $\square \mathrm{kg} / \mathrm{m}^{2} \square$} \\
\hline pre-pregnancy & $24.97 \pm 3.21$ & $24.36 \pm 4.06$ & - & 0.142 \\
\hline antepartum & $29.43 \pm 3.75$ & $28.66 \pm 4.23$ & - & 0.090 \\
\hline Weight gain (kg) & $19.90 \pm 11.29$ & $19.80 \pm 11.04$ & - & 0.864 \\
\hline Mode of delivery, No. (\%) & & & 1.884 & 0.597 \\
\hline Spontaneous vaginal & $101(64.7)$ & $103(66.0)$ & & \\
\hline Assisted vaginal & $13(8.3)$ & $10(6.4)$ & & \\
\hline Elective cesarean & $24(15.4)$ & $30(19.3)$ & & \\
\hline Emergency cesarean & $18(11.6)$ & $13(8.3)$ & & \\
\hline \multicolumn{5}{|l|}{ Neonatal outcomes } \\
\hline Gender, No. (\%) & & & 0.633 & 0.426 \\
\hline Male & $89(57.1)$ & $81(51.9)$ & & \\
\hline Female & $67(42.9)$ & $75(48.1)$ & & \\
\hline Birth weight (g) & $3141.24 \pm 312.88$ & $3124.23 \pm 388.84$ & - & 0.477 \\
\hline Head circumference $\llbracket \mathrm{cm} \square$ & $32.67 \pm 2.10$ & $32.74 \pm 2.03$ & - & 0.722 \\
\hline Fetal length $\square \mathrm{cm} \square$ & $48.53 \pm 2.00$ & $48.39 \pm 2.05$ & - & 0.536 \\
\hline Neonatal hypoglycemia, No. (\%) & $21(13.5)$ & $3(2.0)$ & 298.076 & $<0.001 *$ \\
\hline Meconium-stained amniotic fluid, No. (\%) & & & 2.992 & 0.393 \\
\hline Clear & $98(62.8)$ & $112(71.8)$ & & \\
\hline I & $46(29.5)$ & $34(21.8)$ & & \\
\hline II & $9(5.8)$ & $8(5.1)$ & & \\
\hline III & $3(1.9)$ & $2(1.3)$ & & \\
\hline \multicolumn{5}{|l|}{ Bad outcomes after delivery, No. (\%) } \\
\hline Apgar $\leq 7$ at $1 \mathrm{~min}$ & $5(3.2)$ & $7(4.5)$ & 0.087 & 0.768 \\
\hline Apgar $\leq 7$ at $5 \mathrm{~min}$ & $1(0.6)$ & $1(0.6)$ & - & - \\
\hline Apgar $\leq 7$ at $10 \mathrm{~min}$ & $1(0.6)$ & $1(0.6)$ & - & - \\
\hline Admission to intensive care unit & $3(1.9)$ & $1(0.6)$ & 0.253 & 0.615 \\
\hline
\end{tabular}

Data given as the mean \pm SD or No.(\%). GDM: gestational diabetes mellitus; BMI: body mass index; a:glycosylated hemoglobin monitoring range is $5.5-6.4 \%$; *:significant difference 


\begin{tabular}{|c|c|c|c|c|}
\hline & All groups & $\mathrm{GDM}^{\mathrm{a}}$ & non-GDM & $P$ \\
\hline \multicolumn{5}{|l|}{$\overline{\mathrm{OGTT}} \rrbracket \mathrm{mmol} / \mathrm{L} \square$} \\
\hline Fasting blood glucose & $4.46 \pm 0.68$ & $4.83 \pm 0.57$ & $4.09 \pm 0.57$ & $\stackrel{<}{<}$ \\
\hline \multirow[t]{2}{*}{ 1-hour postprandial blood glucose } & $9.47 \pm 0.94$ & $9.86 \pm 0.80$ & $9.08 \pm 0.90$ & $<$ \\
\hline & & & & $0.001^{*}$ \\
\hline \multirow[t]{2}{*}{ 2-hour postprandial blood glucose } & $7.54 \pm 1.35$ & $7.65 \pm 1.44$ & $7.44 \pm 1.25$ & $<$ \\
\hline & & & & $0.001^{*}$ \\
\hline \multirow[t]{2}{*}{$\begin{array}{l}\text { Glycosylated haemoglobin (24-28 } \\
\text { weeks, \%) }\end{array}$} & $5.51 \pm 0.71$ & $5.97 \pm 0.27$ & $5.06 \pm 0.73$ & $<$ \\
\hline & & & & $0.001 *$ \\
\hline \multirow[t]{2}{*}{$\begin{array}{l}\text { Glycosylated haemoglobin (before } \\
\text { delivery, \%) }\end{array}$} & $5.59 \pm 0.56$ & $5.96 \pm 0.24$ & $5.21 \pm 0.53$ & $<$ \\
\hline & & & & $0.001^{*}$ \\
\hline \multicolumn{5}{|c|}{ Plasma lipid test ((before delivery, mmol/L) } \\
\hline TG & $4.06(2.98,5.41)$ & $4.54(3.23,6.19)$ & $3.80(2.84,5.02)$ & $\stackrel{<}{<}$ \\
\hline TC & $6.21(5.34,6.94)$ & $6.43(5.40,6.98)$ & $6.01(5.30,6.91)$ & 0.200 \\
\hline HDL-C & $1.73(1.49,2.05)$ & $1.71(1.51,2.00)$ & $1.78(1.47,2.11)$ & 0.526 \\
\hline LDL-C & $2.89(2.33,3.43)$ & $2.99(2.41,3.53)$ & $2.86(2.25,3.40)$ & 0.228 \\
\hline non-HDL-C & $4.41(3.67,5.04)$ & $4.57(3.86,5.10)$ & $4.30(3.55,4.92)$ & $0.046^{*}$ \\
\hline sdLDL-C & $1.45(1.14,1.76)$ & $1.48(1.19,1.80)$ & $1.43(1.13,1.75)$ & 0.168 \\
\hline \multicolumn{5}{|l|}{ Cord blood test $(\mu \mathrm{M} \square$} \\
\hline Ala & $\begin{array}{c}212.12(158.64 \\
300.96)\end{array}$ & $\begin{array}{c}212.12(158.99 \\
311.02)\end{array}$ & $\begin{array}{c}211.91(157.55 \\
285.07)\end{array}$ & 0.700 \\
\hline Arg & $2.22(1.57,3.64)$ & $2.20(1.56,3.76)$ & $2.36(1.57,3.41)$ & 0.849 \\
\hline Asp & $\begin{array}{c}26.52(18.62 \\
37.07)\end{array}$ & $\begin{array}{l}26.78(19.01 \\
37.99)\end{array}$ & $\begin{array}{c}26.44(17.57 \\
35.20)\end{array}$ & 0.338 \\
\hline Cit & $9.05(7.59,11.11)$ & $8.95(7.64,11.03)$ & $9.19(7.55,11.41)$ & 0.703 \\
\hline Gln & $2.80(1.62,8.65)$ & $2.85(1.60,8.34)$ & $2.66(1.62,8.99)$ & 0.694 \\
\hline Glu & $\begin{array}{c}370.30(292.42 \\
477.31)\end{array}$ & $\begin{array}{c}369.99(297.3 \\
481.85)\end{array}$ & $\begin{array}{c}372.21(285.83 \\
477.31)\end{array}$ & 0.920 \\
\hline Gly & $\begin{array}{c}473.96(325.21 \\
651.33)\end{array}$ & $\begin{array}{c}594.19(373.95 \\
752.6)\end{array}$ & $\begin{array}{c}407.45(294.6 \\
545.38)\end{array}$ & $\begin{array}{c}< \\
0.001^{*}\end{array}$ \\
\hline His & $\begin{array}{c}21.34(16.46 \\
27.83)\end{array}$ & $\begin{array}{c}22.22(16.83 \\
27.87)\end{array}$ & $\begin{array}{c}20.73(16.27 \\
27.60)\end{array}$ & 0.230 \\
\hline Leu & $\begin{array}{c}95.22(79.40 \\
116.08)\end{array}$ & $\begin{array}{c}93.72(76.16 \\
113.96)\end{array}$ & $\begin{array}{c}96.46(80.15 \\
118.97)\end{array}$ & 0.290 \\
\hline Met & $\begin{array}{c}23.61(19.35 \\
28.16)\end{array}$ & $\begin{array}{c}23.65(19.14 \\
27.66)\end{array}$ & $\begin{array}{c}23.59(19.89 \\
28.26)\end{array}$ & 0.752 \\
\hline Orn & $\begin{array}{c}48.58(37.68 \\
67.63)\end{array}$ & $\begin{array}{c}48.54(36.98 \\
69.18)\end{array}$ & $\begin{array}{c}48.58(38.81 \\
59.95)\end{array}$ & 0.830 \\
\hline Phe & $\begin{array}{c}49.10(41.23 \\
58.94)\end{array}$ & $\begin{array}{c}49.61(41.91 \\
59.16)\end{array}$ & $\begin{array}{c}48.37(40.99 \\
58.53)\end{array}$ & 0.641 \\
\hline Ser & $\begin{array}{c}87.77(68.56 \\
110.18)\end{array}$ & $\begin{array}{c}89.12(69.82 \\
118.46)\end{array}$ & $\begin{array}{c}85.20(68.15 \\
106.52)\end{array}$ & 0.187 \\
\hline Thr & $14.70(11.98$ & $15.14(12.41$ & $14.40(11.81$ & 0.093 \\
\hline
\end{tabular}


18.53)

19.02)

18.00)

\begin{tabular}{|c|c|c|c|c|}
\hline $\operatorname{Trp}$ & $\begin{array}{c}43.13(35.38 \\
53.45)\end{array}$ & $43.51(33.91,53.1)$ & $\begin{array}{c}42.91(35.83 \\
54.33)\end{array}$ & 0.860 \\
\hline Tyr & $\begin{array}{c}61.76(51.78 \\
76.71)\end{array}$ & $\begin{array}{c}63.49(51.82 \\
77.44)\end{array}$ & $\begin{array}{c}60.00(51.55 \\
76.18)\end{array}$ & 0.461 \\
\hline Val & $\begin{array}{c}92.23(77.47 \\
108.50)\end{array}$ & $\begin{array}{c}92.79(76.19 \\
107.87)\end{array}$ & $\begin{array}{c}92.05(78.14 \\
109.28)\end{array}$ & 0.884 \\
\hline Free Carnitine & $\begin{array}{c}24.57(19.37 \\
31.66)\end{array}$ & $\begin{array}{c}25.09(19.31 \\
31.59)\end{array}$ & $\begin{array}{c}24.16(19.37 \\
31.78)\end{array}$ & 0.737 \\
\hline Acetylcarnitine & $\begin{array}{c}16.75(11.65 \\
23.71)\end{array}$ & $\begin{array}{c}16.75(11.65 \\
23.68)\end{array}$ & $\begin{array}{c}16.77(11.41 \\
23.79)\end{array}$ & 0.720 \\
\hline Propionylcarnitine & $1.03(0.69,1.50)$ & $1.05(0.71,1.52)$ & $0.97(0.64,1.47)$ & 0.374 \\
\hline Malonylcarnitine & $0.05(0.03,0.08)$ & $0.05(0.03,0.07)$ & $0.05(0.03,0.08)$ & 0.628 \\
\hline Butylcarnitine & $0.14(0.10,0.18)$ & $0.14(0.10,0.21)$ & $0.13(0.09,0.18)$ & 0.104 \\
\hline Succinylcarnitine & $0.18(0.14,0.25)$ & $0.18(0.13,0.24)$ & $0.19(0.15,0.26)$ & 0.157 \\
\hline 3-Hydroxyisobutyryl-carnitine & $0.11(0.07,0.16)$ & $0.11(0.07,0.16)$ & $0.11(0.07,0.16)$ & 0.764 \\
\hline Isovalerylcarnitine & $0.10(0.08,0.14)$ & $0.11(0.09,0.14)$ & $0.09(0.08,0.13)$ & $0.019^{*}$ \\
\hline Senecioylcarnitine & $0.03(0.02,0.03)$ & $0.03(0.02,0.03)$ & $0.03(0.02,0.03)$ & 0.215 \\
\hline Glutarylcarnitine & $0.04(0.02,0.05)$ & $0.04(0.02,0.06)$ & $0.04(0.02,0.05)$ & 0.538 \\
\hline 3-Hydroxyisovaleryl-carnitine & $0.14(0.11,0.17)$ & $0.15(0.12,0.18)$ & $0.14(0.11,0.17)$ & $0.037^{*}$ \\
\hline Caproylcarnitine & $0.02(0.01,0.04)$ & $0.02(0.01,0.04)$ & $0.02(0.02,0.04)$ & 0.451 \\
\hline Hexenoylcarnitine & $0.01(0.01,0.01)$ & $0.01(0.01,0.01)$ & $0.01(0.01,0.01)$ & 0.798 \\
\hline Adipoylcarnitine & $0.03(0.02,0.04)$ & $0.03(0.02,0.04)$ & $0.03(0.02,0.04)$ & 0.993 \\
\hline Decoylcarnitine & $0.04(0.02,0.06)$ & $0.04(0.02,0.06)$ & $0.04(0.02,0.06)$ & 0.793 \\
\hline Octenylcarnitine & $0.07(0.05,0.10)$ & $0.07(0.05,0.09)$ & $0.07(0.04,0.10)$ & 0.912 \\
\hline Octanedioylcarnitine & $0.02(0.01,0.02)$ & $0.02(0.01,0.02)$ & $0.02(0.01,0.02)$ & 0.820 \\
\hline Decanoylcarnitine & $0.04(0.02,0.08)$ & $0.04(0.02,0.08)$ & $0.04(0.02,0.08)$ & 0.875 \\
\hline Alkaloidcarnitine & $0.04(0.02,0.06)$ & $0.04(0.02,0.06)$ & $0.04(0.02,0.06)$ & 0.690 \\
\hline Lauroylcarnitine & $0.06(0.04,0.10)$ & $0.06(0.04,0.10)$ & $0.06(0.04,0.09)$ & 0.384 \\
\hline Myrcenecarnitine & $0.02(0.01,0.04)$ & $0.02(0.01,0.04)$ & $0.02(0.01,0.04)$ & 0.671 \\
\hline Myristoylcarnitine & $0.15(0.12,0.20)$ & $0.15(0.12,0.20)$ & $0.15(0.12,0.20)$ & 0.822 \\
\hline Alkenyl-myristoyl- carnitine & $0.05(0.03,0.08)$ & $0.05(0.03,0.09)$ & $0.05(0.03,0.08)$ & 0.645 \\
\hline 3-hydroxy-Myristoyl-carnitine & $0.01(0.01,0.02)$ & $0.01(0.01,0.02)$ & $0.01(0.01,0.02)$ & 0.889 \\
\hline Palmitoylcarnitine & $1.76(1.19,2.46)$ & $1.84(1.16,2.47)$ & $1.71(1.20,2.41)$ & 0.678 \\
\hline Palmitoyl -enoyl-carnitine & $0.10(0.06,0.15)$ & $0.10(0.05,0.14)$ & $0.10(0.06,0.15)$ & 0.655 \\
\hline 3-hydroxy-palmitoyl -carnitine & $0.02(0.01,0.02)$ & $0.02(0.01,0.02)$ & $0.02(0.01,0.02)$ & 0.339 \\
\hline Octadecylcarnitine & $0.65(0.48,0.86)$ & $0.70(0.51,0.88)$ & $0.63(0.46,0.85)$ & 0.192 \\
\hline Octadecenoylcarnitine & $0.96(0.70,1.22)$ & $1.01(0.73,1.28)$ & $0.91(0.68,1.18)$ & 0.099 \\
\hline 3-hydroxy-octadecanoyl-carnitine & $0.01(0.01,0.02)$ & $0.01(0.01,0.02)$ & $0.01(0.01,0.02)$ & 0.924 \\
\hline
\end{tabular}

Data given as the mean \pm SD in maternal glucose profiles and as the median $\left(10^{\text {th }}, 90^{\text {th }}\right)$ in others. Gestational diabetes mellitus, GDM; triglycerides; oral glucose tolerance test, OGTT; triglyceride, TG; total cholesterol, TC; high-density lipoprotein-cholesterol, HDL-C; low-density lipoprotein-cholesterol, LDL-C; non-high-density lipoprotein-cholesterol, non-HDL-C; small and dense low-density lipoprotein-cholesterol, sdLDL-C; alanine, Ala; arginine, Arg; aspartic acid, Asp; Citrulline, Cit; glutamine, Gln; glutamic acid ,Glu; glycine, Gly; histidine, His; leucine, Leu; methionine, Met; ornithine, Orn; phenylalanine, Phe; serine, Ser; threonine, Thr; tryptophan ,Trp; tyrosine, Tyr; valine, Val;a:glycosylated hemoglobin monitoring range is 5.5-6.4\%; *:significant difference 
Table 3 Correlations between Neonatal outcomes and maternal characteristics

\begin{tabular}{lcccc}
\hline & \multicolumn{2}{c}{ Neonatal hypoglycemia } & \multicolumn{2}{c}{ Gestational weeks } \\
\cline { 2 - 5 } & $\boldsymbol{\Gamma}$ & $\boldsymbol{P}$ & $\boldsymbol{\Gamma}$ & $\boldsymbol{P}$ \\
\hline Maternal Age & 0.078 & 0.172 & 0.025 & 0.663 \\
Antepartum BMI & 0.126 & $0.027^{\#}$ & -0.113 & $0.046^{\#}$ \\
\hline GDM $^{\mathrm{a}}$ & 0.217 & $<0.001^{\#}$ & -0.243 & $<0.001^{\#}$ \\
\hline Maternal TG* & 0.062 & 0.278 & -0.105 & 0.064 \\
Maternal non-HDL-C* & 0.008 & 0.891 & 0.010 & 0.864 \\
Maternal GHb* & 0.148 & $0.009^{\#}$ & -0.178 & $0.002^{\#}$ \\
\hline Birth weight & -0.104 & 0.067 & 0.228 & $<0.001^{\#}$ \\
\hline Neonatal hypoglycemia & - & - & -0.119 & $0.036^{\#}$ \\
\hline
\end{tabular}

Gestational diabetes mellitus, GDM; triglyceride, TG; Body mass index, BMI; Glycosylated haemoglobin,GHb; a:glycosylated hemoglobin monitoring range is $5.5-6.4 \%$; *:before delivery; \#:significant difference

Table 4 Correlations between neonate blood tests and maternal-fetal characteristics

\begin{tabular}{lcccccc}
\hline & \multicolumn{2}{c}{ Glycine } & \multicolumn{2}{c}{ Isovalerylcarnitine } & 3-Hydroxyisovaleryl-carnitine \\
\cline { 2 - 7 } & $\boldsymbol{\Gamma}$ & $\boldsymbol{P}$ & $\boldsymbol{r}$ & $\boldsymbol{P}$ & $\boldsymbol{r}$ & $\boldsymbol{P}$ \\
\hline Maternal Age & -0.083 & 0.142 & $<0.001$ & 0.999 & -0.051 & 0.370 \\
Antepartum BMI & 0.146 & $0.010^{\#}$ & 0.011 & 0.844 & -0.045 & 0.428 \\
\hline Gestational weeks & -0.195 & $0.001^{\#}$ & -0.113 & $0.046^{\#}$ & -0.082 & 0.149 \\
\hline GDM & 0.297 & $<0.001^{\#}$ & 0.133 & $0.019^{\#}$ & 0.118 & $0.037^{\#}$ \\
\hline Maternal TG* & -0.027 & 0.629 & 0.047 & 0.412 & -0.067 & 0.240 \\
\hline Maternal non-HDL-C* & 0.089 & 0.116 & -0.029 & 0.611 & 0.002 & 0.978 \\
\hline Maternal GHb* & 0.163 & $0.004^{\#}$ & 0.161 & $0.004^{\#}$ & 0.116 & $0.040^{\#}$ \\
\hline Birth weight & 0.034 & 0.548 & 0.038 & 0.509 & 0.010 & 0.859 \\
\hline Neonatal hypoglycemia & 0.050 & 0.377 & 0.101 & 0.074 & 0.073 & 0.200 \\
\hline
\end{tabular}

Gestational diabetes mellitus, GDM; triglyceride, TG; Body mass index, BMI; Glycosylated haemoglobin,GHb;*:before delivery; \#:significant difference

Table 5 Logistic regression to evaluate the association between maternal variables and neonatal hypoglycemia

$\begin{array}{llllll}\text { Variable } & \text { Coeffificient } & \text { Standard Error } & \text { Simple OR (95\% CI) } & \boldsymbol{P} & \text { Adjusted OR (95\% CI) }\end{array}$

\begin{tabular}{ccccccc}
\hline $\mathrm{GDM}^{\mathrm{a}}$ & 1.75 & 0.81 & $5.77(1.18,28.36)$ & 0.031 & - & - \\
$\mathrm{BMl}^{*}$ & 0.10 & 0.06 & $1.10(0.97,1.23)$ & 0.088 & $1.06(0.93,1.20)$ & 0.373 \\
Ghb $^{*}$ & 0.27 & 0.77 & $1.31(0.29,5.88)$ & 0.725 & $0.18(0.20,1.35)$ & 0.095 \\
Gestational weeks & -0.03 & 0.03 & $0.97(0.92,1.02)$ & 0.198 & $0.97(0.92,1.03)$ & 0.382 \\
\hline
\end{tabular}

GDM: gestational diabetes mellitus; a:glycosylated hemoglobin monitoring range is 5.5-6.4\%; body mass index,BMl;*:before delivery; \#:adjusted by $\mathrm{GDM}^{\mathrm{a}}$ 
Table 6 The relationship between GDM and umbilical cord blood of amino acid and carnitine via ANCOVA analysis

\begin{tabular}{ccccccc}
\hline & \multicolumn{2}{c}{ Glycine } & \multicolumn{2}{c}{ Isovalerylcarnitine } & 3-Hydroxyisovaleryl-carnitine \\
\cline { 2 - 7 } & $\boldsymbol{b}$ & $\boldsymbol{P}$ & $\boldsymbol{b}$ & $\boldsymbol{P}$ & $\boldsymbol{b}$ & $\boldsymbol{P}$ \\
\hline GDM $^{\text {a }}$ & 0.449 & 0.010 & 0.000 & 0.989 & 0.001 & 0.588 \\
BMI $^{*}$ & 0.016 & 0.033 & - & - & - & - \\
Ghb $^{*}$ & 0.783 & 0.669 & 0.535 & 0.960 & 0.019 & 1.000 \\
Gestational weeks & 0.008 & 0.127 & 0.001 & 0.606 & - & - \\
\hline
\end{tabular}

GDM: gestational diabetes mellitus; a:glycosylated hemoglobin monitoring range is 5.5-6.4\%; body mass index,BMI;*:before delivery;

\section{Figures}

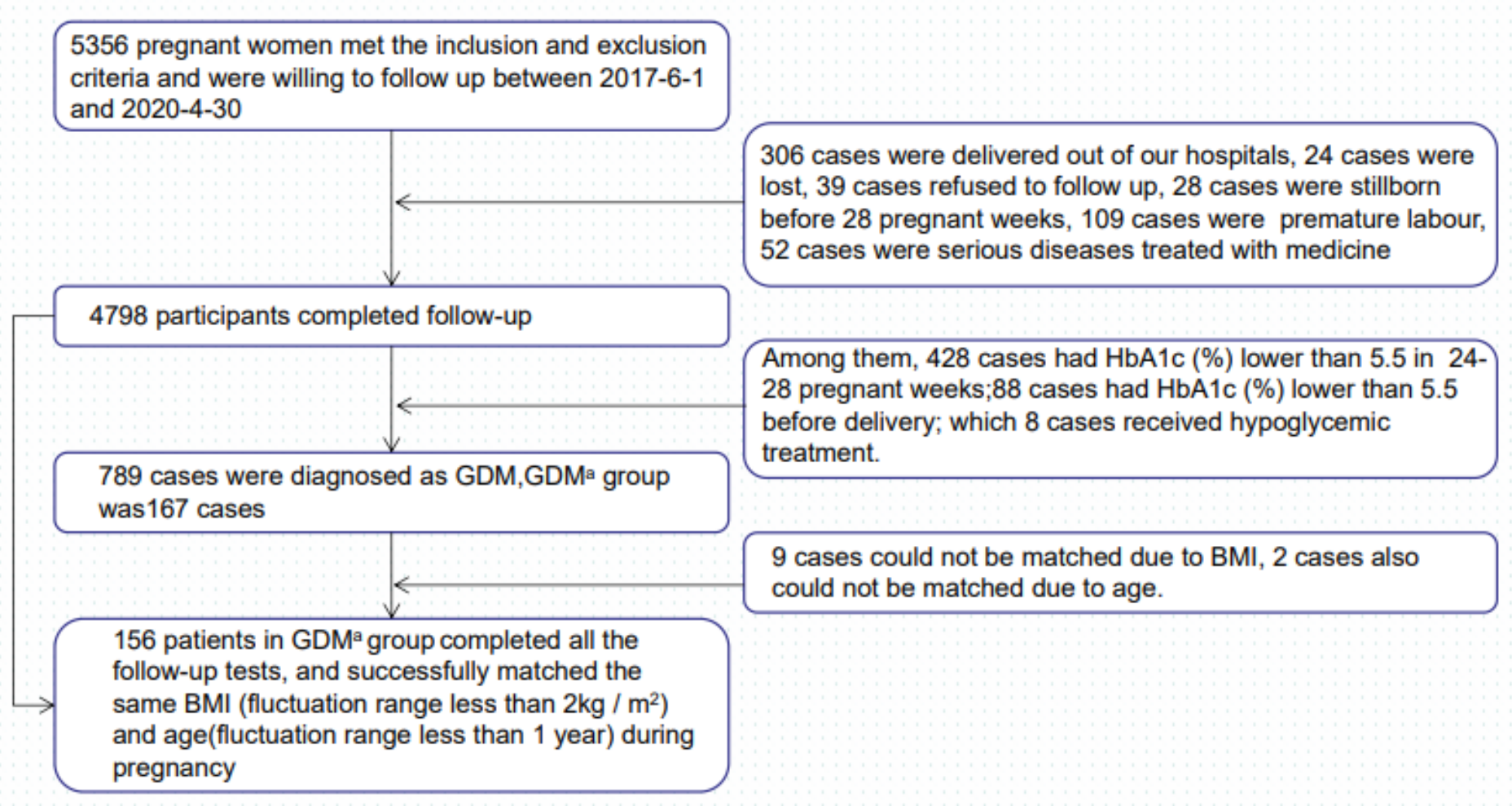

Figure 1 Flow diagram of study inclusion criteria

GDM:Gestational diabetes mellitus;BMI:Body mass index;HbA1c(\%):glycated hemoglobin

\section{Figure 1}

Flow diagram of study inclusion criteria. It shows a series of details on the inclusion and exclusion on the study population

\section{Supplementary Files}

This is a list of supplementary files associated with this preprint. Click to download. 
- AppendixFigure1.pdf

Page 19/19 